Article

\title{
Research and Modeling of Photovoltaic Array Channel Noise Characteristics
}

\author{
Fengjie Sun and Chenkai Zhao * \\ School of Electrical and Electronic Engineering, North China Electric Power University, Changping District, \\ Beijing 102206, China; sfj@ncepu.edu.cn \\ * Correspondence: 1172201444@ncepu.edu.cn; Tel.: +86-010-6177-3964
}

Received: 13 March 2019; Accepted: 29 March 2019; Published: 1 April 2019

\begin{abstract}
The photovoltaic array can be used as a medium for carrier communication to realize monitoring of photovoltaic components. Photovoltaic array channel noise, especially the pulse-type noise therein, seriously interferes carrier communication, so it is necessary to grasp the characteristics of the photovoltaic array channel noise. Photovoltaic array channel noise modeling is a key process when conducting anti-noise immunity tests of monitoring equipment. Based on the time-domain waveform of photovoltaic series channel noise which is measured in a photovoltaic power station, this paper proposes a photovoltaic array noise modeling method of Wavelet Peak-Type Markov chain, and studies the influence on modeling accuracy when different mother wavelets are adopted for modeling. From the simulation results, root mean square errors of the predicted output for Haar, Biorthogonal and Daubechies wavelet-based function modeling case are $0.9614 \mathrm{~V}, 1.4915 \mathrm{~V}$ and $0.7928 \mathrm{~V}$, respectively, validating that Daubechies wavelet-based function is the best wavelet-based function of modeling. In the case that the peak of original noise reaches $20 \mathrm{~V}$, the predicted mean absolute error of this model is only $0.4926 \mathrm{~V}$, which not only verifies the applicability of the Wavelet Peak-Type Markov chain model to the photovoltaic array channel noise, but also verifies the applicability to the pulse-type noise.
\end{abstract}

Keywords: photovoltaic array channel noise; wavelet packet decomposition and recombination; Peak-Type Markov chain; noise characteristics and modeling

\section{Introduction}

With the rapid development of the photovoltaic industry, fault monitoring in photovoltaic power plants has become an important issue because the performance of photovoltaic modules affects the output characteristics of photovoltaic arrays directly, thus further affecting the stability of the photovoltaic generation system [1]. At present, the Chinese power system is actively constructing smart grids and energy big data centers [2-4]. In order to transmit the operating parameters such as voltage, current and temperature of the monitored photovoltaic modules to the big data center, wireless, carrier and many other technologies that are required for data communication. In [5-7], PLC communication technology is applied to the monitoring system of photovoltaic module working conditions. In [8,9], wireless communication methods are applied to the monitoring of the working status of photovoltaic modules. In [10], A single DC wire carrier communication mode is adopted among photovoltaic modules to transmit the operation status data of photovoltaic modules in a photovoltaic power station, which uses the photovoltaic array as a medium for carrier communication without adding extra wiring for communication.

For photovoltaic power plants without isolation transformers, no batteries, and no EMI filters in the DC-side, the inverters will generate severe common-mode and differential-mode interference among photovoltaic arrays channel when they are connected to the grid [11-14]. Therefore, it is 
necessary to grasp the channel noise characteristics of the photovoltaic array, laying a foundation for ensuring the progress of carrier communication among photovoltaic arrays. References [15-17] introduce in detail the propagation path and generation mechanism of conducted electromagnetic interference (EMI) in PWM inverter systems, modeling and analyzing EMI. Based on these studies, we study the electromagnetic interference on the DC-side of the photovoltaic inverter and construct a simplified electromagnetic interference circuit model of the photovoltaic power generation system. At present, there are few studies on the noise characteristics of photovoltaic array channels, and more are focused on the measurement and modeling of noise in AC power line channels. Therefore, it is necessary to set up an experimental platform for real-time measurement and analysis of photovoltaic array channel noise interference.

According to measurements, photovoltaic array channel noise, especially the pulse-type noise therein, seriously interferes with carrier communications. However, due to the lack of a reliable analog noise source which accords with the relevant standard, at present, the current anti-noise immunity tests of related monitoring equipment still have difficulties. Therefore, it is necessary to conduct in-depth studies on power line noise modeling technology, which has important theoretical and engineering significance. Noise modeling needs to refer to the modeling method of AC power line channel noise. In [18], an AR autoregressive model is established for low-voltage AC power line channel background noise, but AR models are not suitable for pulse-type noise analysis in photovoltaic array channels. Li et al. measured the noise of AC power line channels in China and Italy, modeling the pulse-type noise in these two countries using two models, a Middleton Class A (MCA) model and a stable distribution model [19]. In [20], a Peak-Type Markov chain modeling method is proposed, which is suitable for the modeling of background noise and impulse noise, but there is still a certain gap between the model output and the original noise in the frequency domain characteristics obtained by simulation; In another study [21], a low-voltage power line background noise Wavelet-Markov chain modeling method was proposed. This method improves the insufficiencies of Peak-Type Markov model. The wavelet packet decomposition algorithm is introduced to optimize the model, and the predicted background noise has a good fit to the original noise in the time domain and the frequency domain, but this reference reported no research on the pulse-type noise.

In order to solve the problem of modeling the mass pulse-type noise in photovoltaic array channel noise, this paper proposes a method of Wavelet Peak-Type Markov chain, and studies the influence on modeling accuracy when different mother wavelets are used for modeling, based on the time-domain waveform of photovoltaic array channel noise which is measured in photovoltaic power station. The noise model can accurately fit the time-domain waveform of the periodic pulse-type noise and the background noise in the photovoltaic array channel, providing a theoretical basis for studying the noise characteristics of photovoltaic array channels.

\section{The Measurement of Photovoltaic Array Channel Noise}

\subsection{Noise Measurement Environment}

The experimental platform 1 was built in the outdoor photovoltaic power station of North China Electric Power University in Beijing, China and the photovoltaic array channel noise in the photovoltaic power station was measured to verify the applicability of the proposed noise model for modeling the photovoltaic array channel noise. The measurement environment is shown in Figure 1a. The PV power plant inverter system has no EMI filter on the DC-side.

The experimental platform 2 was built at the Yunjiakou Photovoltaic Power Station located in Qinghai, China. Similarly, the photovoltaic array channel noise was measured to verify the applicability of the noise model for modeling the photovoltaic array channel noise. The measurement environment is shown in Figure 1b. The PV power plant inverter system includes an EMI filter on the DC-side. 

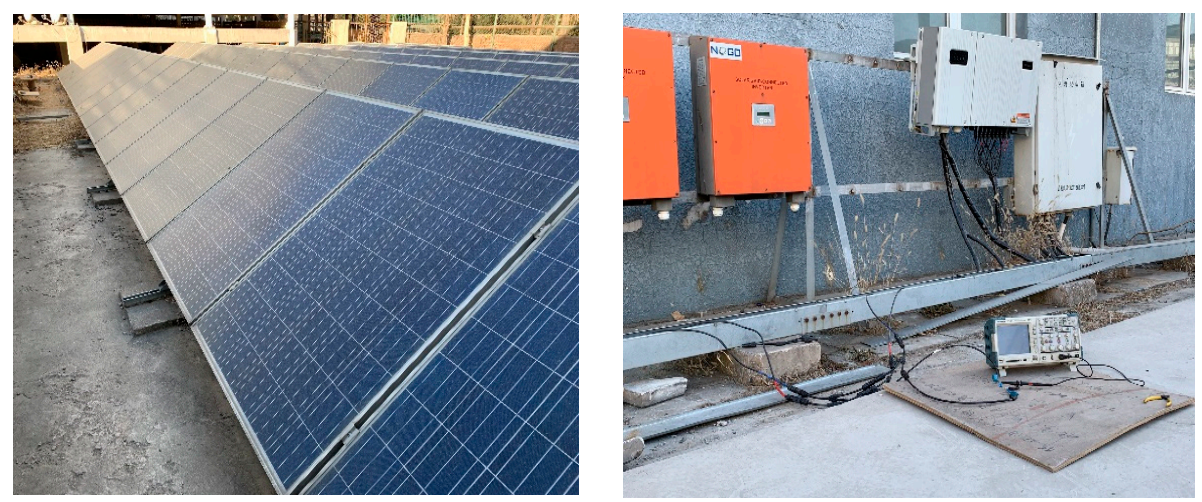

(a)
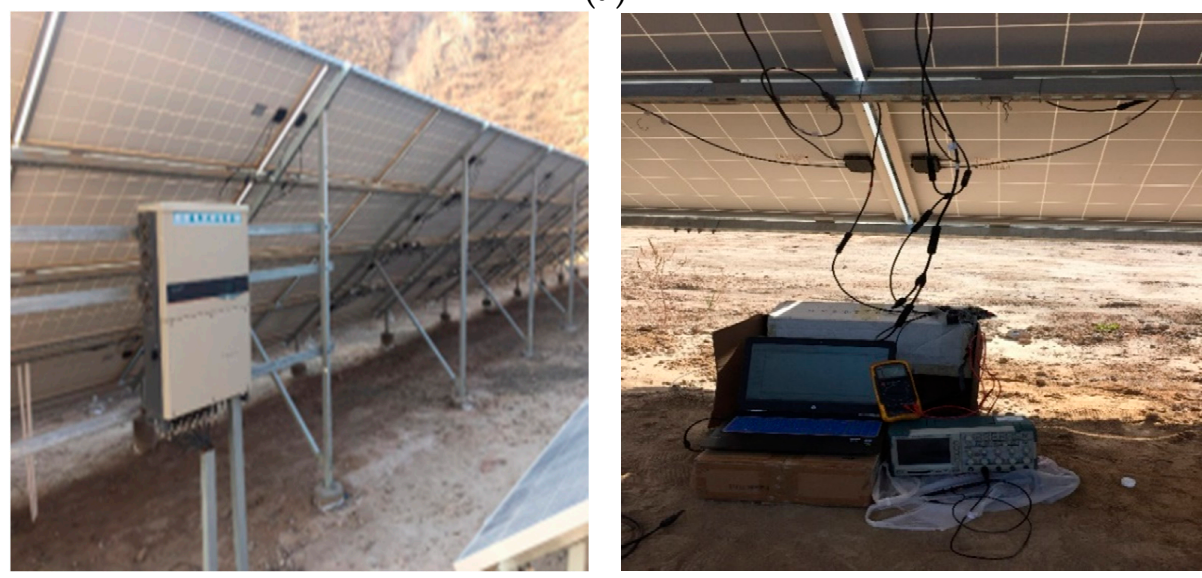

(b)

Figure 1. Photovoltaic series channel noise measurement environment. (a) Experimental platform 1 noise measurement environment; (b) Experimental platform 2 noise measurement environment.

\subsection{Photovoltaic Array Channel Noise Measurement Scheme}

The photovoltaic array channel noise measurement scheme is shown in Figure 2. The measurement circuit is connected in parallel between the PV module series and the inverter. Under normal illumination and temperature, the signal in the PV array passes through a high-pass type resistance-capacitance noise measurement circuit (also known as the impedance stability network LISN [22]). A TPS 2014 digital storage oscillograph (Tektronix, Beaverton, OR, USA) is used to collect noise signals on the outer side of the resistance, store them and then transmit them to the computer for drawing and analysis with MATLAB 2014b (The MathWorks, Inc, Natick, MA, USA).

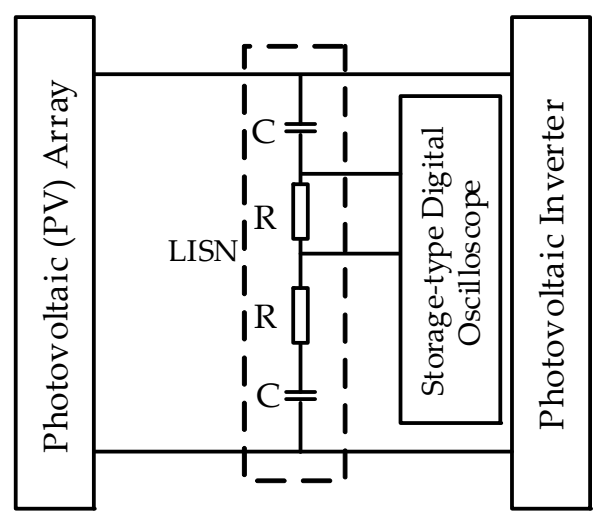

Figure 2. DC-side noise measurement scheme of photovoltaic power station inverter. 


\subsection{Photovoltaic Array Channel Noise Measurement Results}

In the experiment, the noise time-domain waveforms of multiple sets of PV arrays are measured, under the condition that the resistance value of the LISN circuit is $2 \mathrm{k} \Omega$ and the capacitance value is $1 \mu \mathrm{F}$. The typical time-domain waveform of noise which is displayed in the digital storage oscillograph is shown in Figure 3a,b. It should be noted that in Figure 3a, the result of experimental platform 1 is shown in oscilloscope channel $2(\mathrm{CH} 2)$, which has $10 \times$ attenuation compared with the original noise (which means the data observed in Figure 3a needs to be multiplied by 10 to get the real data). The data of typical noise time-domain waveform is imported into MATLAB 2014b for plotting and analysis. The experimental platform measurement results of the outdoor photovoltaic power station built in Beijing North China Electric Power University are shown in Figure 3c. The measurement results of the experimental platform built ate the Fengjiakou Photovoltaic Power Station are shown in Figure 3d.

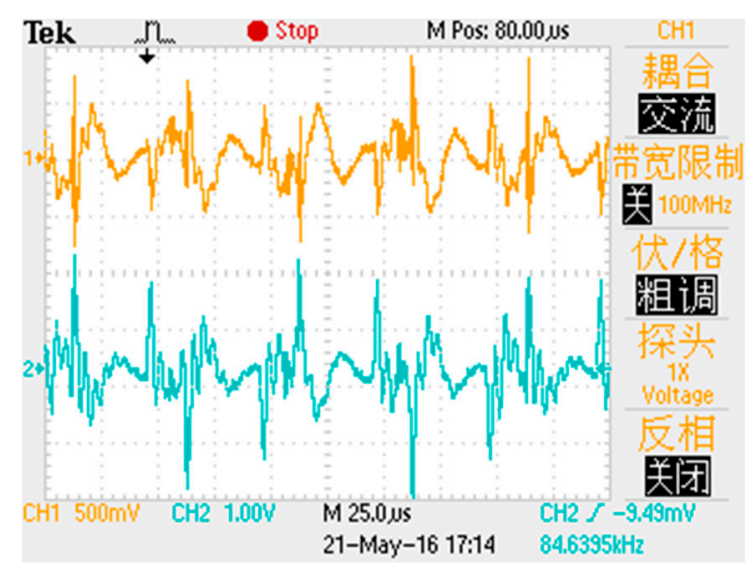

(a)

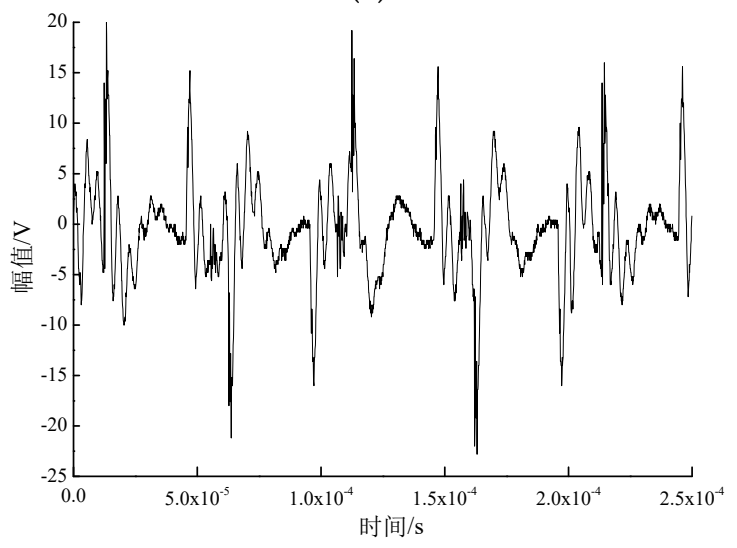

(c)

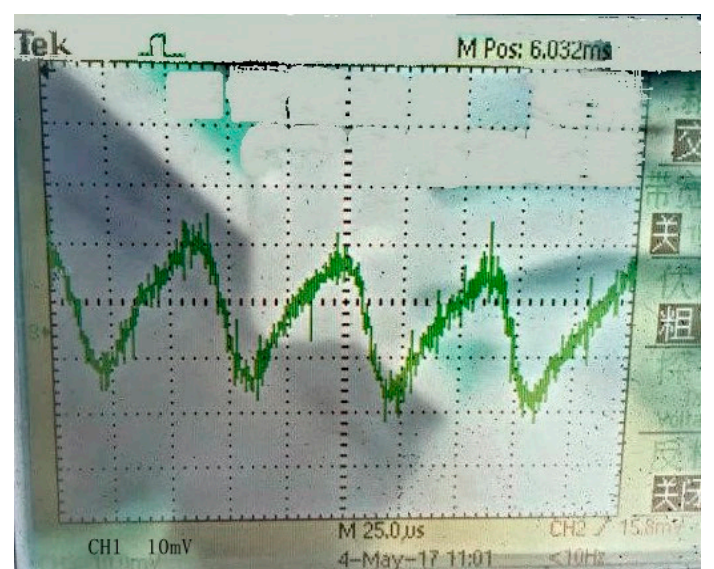

(b)

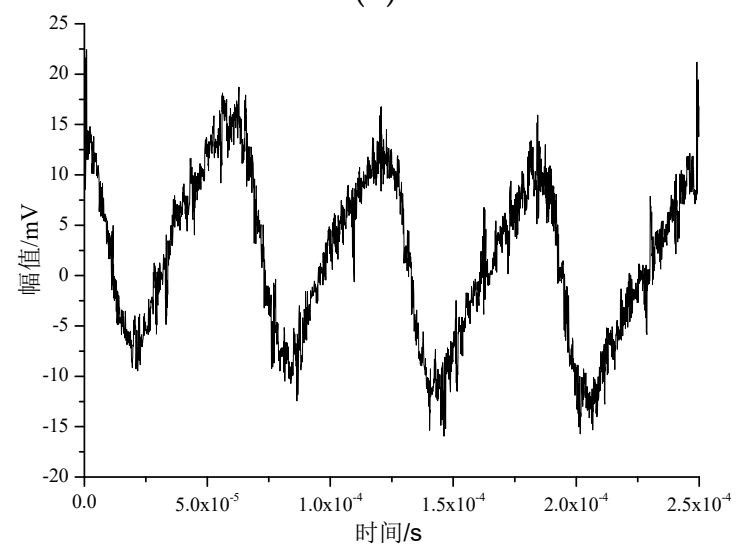

(d)

Figure 3. The time-domain waveform of the measured PV array noise. (a) The measured noise which is observed from the oscillograph for experimental platform $1 ;(\mathbf{b})$ The measured noise which is observed from oscillograph in experimental platform 2; (c) The measured noise in experimental platform 1; (d) The measured noise in experimental platform 2.

\section{Noise Characteristics of Photovoltaic Array Channel}

The experimental results show that there is mass periodic pulse-type noise with high amplitude in the PV array channel when the PV inverter is working. There is always some background noise with low amplitude and high frequency, which is superimposed on the impulse noise.

Photovoltaic power plants have a wide variety of interference factors and complex environments. After analysis, the noise interference signals of photovoltaic arrays are mainly generated by photovoltaic modules and inverters. 


\subsection{Grid and Load, Inverter and Photovoltaic Array Equivalent Circuit Model}

The parallel system of photovoltaic inverters is given by [23]. In order to simplify the analysis of the electromagnetic interference between the inverter and the PV array, we ignore the series resistance and shunt resistance of the PV module equivalent circuit in [1]. When two pairs of bridge arms of a three-phase voltage bridge inverter are alternately turned on and off, the simplified equivalent circuit of the grid and load, inverter and PV array is shown in Figure 4.

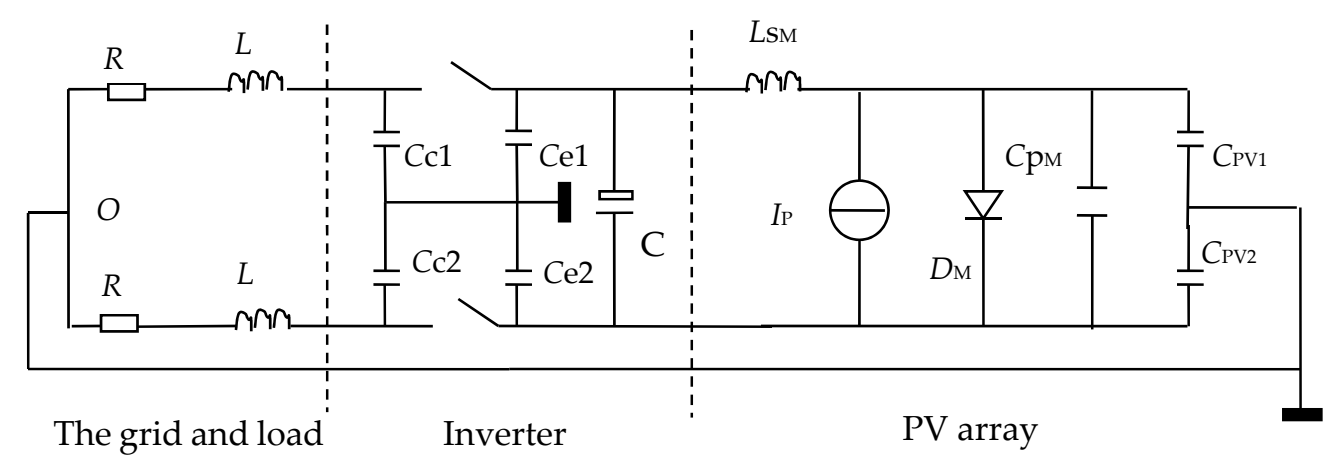

Figure 4. The simplified equivalent circuit for grid and load, inverter and PV module arrays.

In the circuit on the left side of Figure $4, L$ is the equivalent inductance, including the filter inductor on the grid and load side, the parasitic parameters of the transmission line from the inverter to the grid; $R$ is the equivalent load resistance of the load, transmission conductor, etc.; $C$ is the capacitance of the reduced ripple interference in the inverter; $C \mathrm{c} 1, C \mathrm{e} 1, C_{\mathrm{c}} 2$ and $C \mathrm{e} 2$ are the earth equivalent capacitance of collector and emitter of inverter switching devices, respectively. The collector, the emitter and the metal heat sink of the inverter switch tube are coated with a thin thermal grease insulating layer, meanwhile, the heat sink and the equipment casing are screwed together and connected to the ground, thus forming the equivalent ground capacitance among the collector, emitter and the heat sink of the switching transistor. The right side of Figure 4 is the equivalent circuit of the photovoltaic array [24], $C_{\mathrm{PV} 1}$ and $C_{\mathrm{PV} 2}$ are the equivalent capacitance to the ground on both sides of the photovoltaic array; $C \mathrm{p}_{\mathrm{M}}$ is the total equivalent parasitic junction capacitance of the photovoltaic array; $L s_{\mathrm{M}}$ is the total equivalent parasitic inductance of the photovoltaic array; Ip is the output current of the component array; $D_{\mathrm{M}}$ is the equivalent diode of the PV array.

\subsection{Inverter Noise Interference Analysis}

Whether it is an isolated inverter or a non-isolated inverter, the first stage of the inverter is DC/AC conversion. Using PWM modulation technology, the output voltage is a series of PWM pulse voltages, generated by the power switch tube (IGBT, MOSFET, etc.). During the turn-on of the inverter switching device, the inverter and the grid are low-impedance with respect to the PV array, and the PV array will flow to the inverter in the form of an impact spike current, generating an impact peak current, meanwhile, the voltage will drop rapidly, according to Ohm's law.

During the turn-off of the inverter switching device (the previous group of switches is turned off by conduction, the period of the latter group is not turned on, this period is called 'dead time'), the inverter and the grid change the impedance of the PV array from low impedance to high impedance. According to Ohm's law, the voltage will rise rapidly and the peak voltage will be generated, thus the current will drop rapidly [25].

These spike voltages act on the DC bus end, forming conductive differential mode interference to the photovoltaic array channel. Moreover, these interference voltage signals also contain a great number of harmonics under the influence of various equivalent parasitic parameters. Although a large electrolytic capacitor $C$ of several thousand $\mathrm{uF}$ is used to filter out these harmonics, it has a large equivalent resistance and parasitic inductance, and the electrolytic capacitor has a failure effect 
under the circumstance of high (it has been pointed out in the literature that when the electrolytic capacitor is under a frequency of $20 \mathrm{kHz}$, the capacity failure is more than $40 \%$ [26]). Therefore, the electrolytic capacitor cannot filter out these harmonics completely and its function of preventing voltage mutation is greatly reduced. Inductance $L$ also has a large equivalent resistance and parasitic capacitance, and will resonate with other parasitic parameters in the circuit. At the same time, the current flowing through inductance $L$ cannot be mutated, which can delay the rise and fall of peak current to some extent.

When the inverter switching device is turned on, the collector equivalent capacitance $C_{c}$ and Ce of the switching device are connected in parallel to $C$, the voltage across the equivalent capacitor $C$ cannot be abruptly changed, and the current in the filtered equivalent inductor $L$ of the inverter output side cannot be abruptly changed. The peak current generates a reverse induced electromotive force, flowing into the earth through the equivalent capacitor $C$, forming a common-mode interference current between the array and the inverter through $C_{\mathrm{PV} 1}$ and $C_{\mathrm{PV} 2}$, the equivalent capacitances of the PV array to ground.

When the inverter switching device is turned off, as described above, the 'dead time' in the switching circuit of the inverter can form a peak voltage. The peak voltage flows into the ground through $C$, the equivalent capacitance of the inverter to ground, and the common-mode interference current between the component series and the inverter is also formed by the equivalent capacitances $C_{\mathrm{PV} 1}$ and $C_{\mathrm{PV} 2}$, forming common-mode interference between the PV array and inverter.

\subsection{PV Module Noise Interference Analysis}

In the power generation process, the photovoltaic array is affected by environmental factors such as light, temperature, wind direction and wind force, and the photogenerated current changes within a certain frequency range. The interaction of photogenerated currents with varying frequency and amplitude and various non-linear factors present in photovoltaic modules (equivalent diode junction capacitor $C \mathrm{p}$ in the photovoltaic module, equivalent inductance Ls connected to the cell chip and equivalent capacitance $\mathrm{C}_{\mathrm{PV}}$ of the photovoltaic module to the ground, etc) can produce a certain amount of non-linear interference signals.

The measurement results show that the amplitude of the nonlinear interference signal and the electromagnetic interference signal generally does not exceed $-10 \mathrm{dBm}$ when the PV module series has pure resistive load and the inverter is not working. The amplitude of noise interference below $150 \mathrm{kHz}$ is relatively large. The noise interference amplitude is smaller and evenly distributed from $150 \mathrm{kHz}$ to $2 \mathrm{MHz}$, and the interference to carrier communication is small. As long as the amplitude of the carrier communication is sufficiently high and the frequency band with a small interference signal is selected, the normal communication of the carrier is generally not affected.

However, when the inverter is working, the output current of the photovoltaic arrays will instantaneously generate a differential-mode surge current of nearly $10 \mathrm{~A}$ when the inverter switching device is turned on, and will be in the series of photovoltaic components when the inverter switching device is turned off. A certain pulse-type voltage interference signal is formed, which is a very large differential-mode interference signal for carrier communication. Through the two pairs of ground equivalent capacitors $C_{\mathrm{PV} 1}, C_{\mathrm{PV} 2}$ and ground in the series of photovoltaic modules, the photovoltaic array will be coupled to the common-mode current interference signal of the grid and the inverter.

In summary, the photovoltaic module and the inverter are noise interference sources, while the voltage and current type interference signals coexist, and the differential-mode interference and the common-mode interference coexist. These severe noise interference signals are catastrophic for DC single-conductor carrier communication between PV modules.

\subsection{The Electromagnetic Interference Model of Inverter and Photovoltaic Array}

According to the above analysis, the noise interference signal model generated when the inverter switching device is working is shown in Figure 5. 


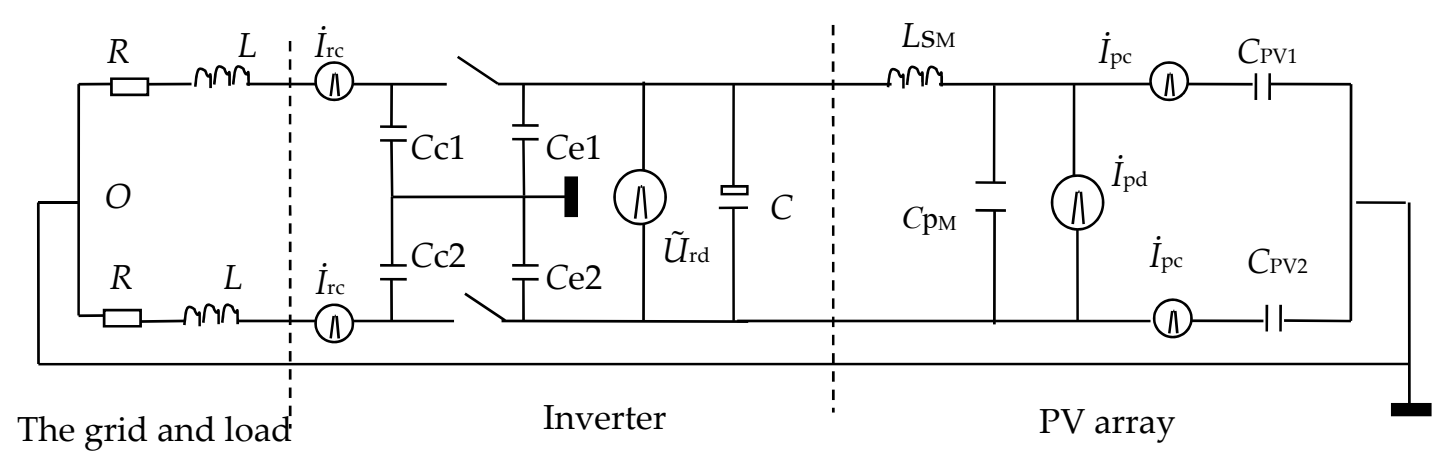

Figure 5. Noise interference model of the inverter and PV array.

In the figure, $L$ is the equivalent inductance, including the filter inductor on the grid and load side, the parasitic parameters of the transmission line from the inverter to the grid; $R$ is the equivalent load resistance of the load, transmission conductor, etc.; $C$ is the capacitance of the reduced ripple interference in the inverter; $\mathrm{Cc} 1, \mathrm{Ce} 1, \mathrm{Cc} 2$ and $\mathrm{Ce} 2$ are the earth equivalent capacitance of collector and emitter of inverter switching devices, respectively; $C_{\mathrm{PV} 1}$ and $C_{\mathrm{PV} 2}$ are the equivalent capacitance to the ground on both sides of the component array; $C \mathrm{p}_{\mathrm{M}}$ is the total equivalent parasitic junction capacitance of the photovoltaic array; $L s_{M}$ is the total equivalent parasitic inductance of the photovoltaic array; Ip is the output current of the component array; $D_{\mathrm{M}}$ is the equivalent diode of the PV array; $I_{\mathrm{rc}}$ is the common-mode current interference signal generated by the inverter; $U_{\mathrm{rd}}$ is the differential-mode voltage interference signal generated by the inverter; $I_{\mathrm{pc}}$ is the common-mode current interference signal generated by the PV array; and $I_{\mathrm{pd}}$ is the differential-mode current interference signal generated by the PV array.

The above analysis is only for the case where the photovoltaic grid-connected power generation system adopts non-isolated type. When the photovoltaic grid-connected power generation system uses an isolation transformer between the inverter and the grid, the common-mode current interference signal will not exist due to the isolation effect of the transformer. However, the differential-mode interference signal will still exist, thus there is still serious interference to the carrier communication.

\section{Wavelet Peak-Type Markov Chain Model}

Observed from the measurement results, there is a large amount of periodic, pulse-type noise in the photovoltaic array channel. For periodic pulse type noise, a wavelet peak-type Markov chain algorithm can be used for modeling. In [27], a peak-type Markov algorithm is proposed for modeling periodic pulse-type noise. The effectiveness of the peak Markov model for impulsive noise modeling has been certified in this reference. The literature [21] proposes a wavelet-Markov chain modeling method for the background noise of AC-side power line channel, the optimization of peak-type Markov chain model is made to solve the shortcomings of the peak-type Markov model that requires multiple noise parameters for modeling. Therefore, this paper will study the application of wavelet peak-type Markov chain in the modeling of photovoltaic array channel noise and the influence of the selection of different wavelet-based functions on the prediction accuracy of noise model.

\subsection{Peak-Type Markov Chain}

Peak-type Markov chain pulse-type noise modeling is divided into an amplitude rising state and an amplitude falling state, that is, a probability transfer matrix of two states needs to be constructed. For the peak-type Markov stochastic process $X(n)$ whose state and time parameters are discrete, if at $k+1$, the $X(k+1)$ state is not only related to $X(k)$, but also $X(k), X(k-1)$ related: when $X(k)$ is greater than $X(k-1)$, the probability that $X(k+1)$ is greater than $X(k)$ is greater than the probability that $X(k+1)$ is less than $X(k)$; When $X(k)$ is less than $X(k-1)$, the probability that $X(k+1)$ is less than $X(k)$ is greater 
than the probability that $X(k+1)$ is greater than $X(k)$ [28]. The transition probability of $X(n)$ satisfies the following conditions:

$$
P\{X(n)=j \mid X(n-1)=i\}=\left\{\begin{array}{l}
P_{1}\{X(n)=j \mid X(n-1)=i\} X(n-1) \geq X(n-2) \\
P_{2}\{X(n)=j \mid X(n-1)=i\} X(n-1)<X(n-2)
\end{array}\right.
$$

It is assumed that the random process $X(n)$ has $N$ states, where $i$ and $j$ are the states in which the random process $X(n)$ may occur, and $P_{1}$ and $P_{2}$ are the transition probabilities of the rising state and the falling state respectively, which constituting the $N \times N$ probability transfer matrices $P_{1}, P_{2}$ of the rising state and the falling state respectively. The probability vector $\Pi(k)$ of the $k$-th transition state is:

$$
\prod(k)= \begin{cases}\prod(k-1) \mathrm{P}_{1} & X(k-1) \geq X(k-2) \\ \prod(k-1) \mathrm{P}_{2} & X(k-1)<X(k-2)\end{cases}
$$

In the formula, $\Pi(k)$ is the probability that each state appears after the $k$-th transition, and the state with the highest probability of selection is the predicted value. This kind of special Markov chain is called peak-type Markov chain, which is often used to solve the pulse-type noise modeling problem, but the peak-type Markov chain has a great problem in solving the periodic pulse-type noise modeling problem [29]. There are limitation: the amplitude rising state group cannot be transferred to the amplitude falling state group, and the amplitude falling state group cannot be transferred to the amplitude rising state group. There are a great number of periodic pulse-type noises in the photovoltaic array channel. For this reason, the wavelet packet transform is introduced in this paper. The original noise signal is firstly decomposed into multiple layers to obtain the wavelet packet coefficient group with non-periodic pulse type properties, and then Each group of wavelet packet coefficients is subjected to peak-type Markov chain modeling to output new wavelet packet coefficients, and finally the new wavelet packet coefficients are reconstructed to obtain predicted output noise.

\subsection{Wavelet Packet Decomposition and Reconstruction}

The wavelet packet transform is an accurate signal analysis method. It can represent the local characteristics of signals in the time-frequency domain and has strong analysis ability for non-stationary signals. The decomposition process is to make the signal through a series of filters with different center frequencies but the same bandwidth, simultaneously decomposing the high and low frequency parts of the signal to improve the frequency resolution [30,31]. The discretized signal sequence is decomposed into layer wavelet packets to obtain sub-signals. The specific decomposition algorithm is shown in Equation (3):

$$
\left\{\begin{array}{c}
f_{2 m}^{j}(n)=\sum_{k \in Z} a(k-2 n) f_{m}^{j-1}(k), k=1,2, \cdots, j \\
f_{2 m+1}^{j}(n)=\sum_{k \in Z} b(k-2 n) f_{m}^{j-1}(k), k=1,2, \cdots, j
\end{array}\right.
$$

where, $f_{m}^{j}(n)$ is the $m$-th decomposition sequence of signal sequence $f(n)$ obtained through $j$-layer decomposition, and $a$ and $b$ are low-pass and high-pass decomposition filter coefficients, respectively. Taking three-layer decomposition as an example, Figure 6 shows the process of decomposition. 


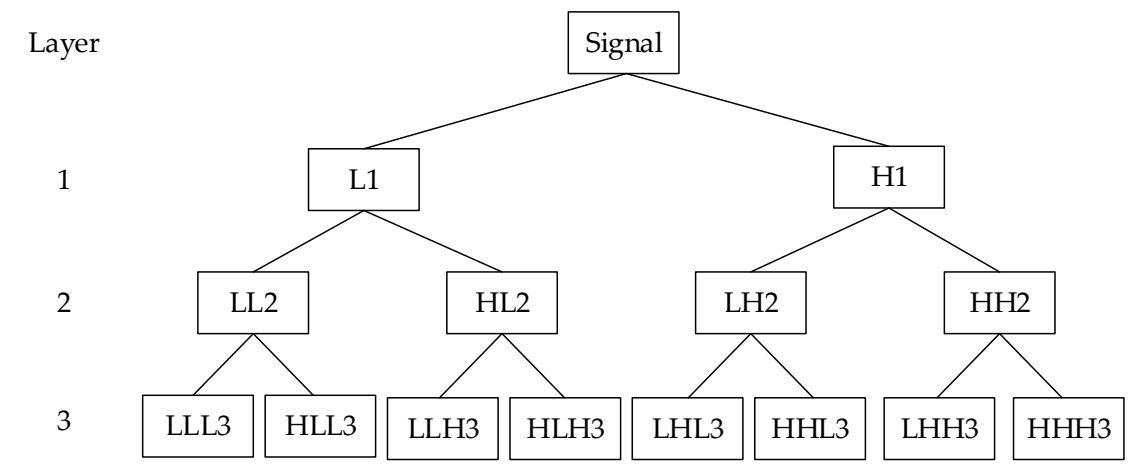

Figure 6. Wavelet packet 3-layer decomposition tree.

The process of reconstructing a series of wavelet packet coefficients into the original signal is called wavelet-packet reconstruction. The specific reconstruction algorithm is as follows:

$$
f_{m}^{j-1}(n)=\sum_{k \in Z} f_{2 m}^{j}(k) c(n-2 k)+\sum_{k \in Z} f_{2 m+1}^{j}(k) d(n-2 k)
$$

where, $c$ and $d$ are low-pass and high-pass reconstruction filter coefficients respectively [32]. Finally, the original signal sequence is reconstructed.

\subsection{Wavelet-Based Functions}

For $\Psi(t) \in L^{2}(R)$, if the condition as shown in Equation (5) is satisfied, it is called a mother wavelet:

$$
\begin{gathered}
C_{\psi}=\int_{-\infty}^{+\infty}|\omega|^{-1}|\psi(\omega)|^{2} \mathrm{~d} \omega<\infty \\
\int_{-\infty}^{+\infty} \psi(t) \mathrm{d} t=0
\end{gathered}
$$

The wavelet packet transform is different from the Fourier transform, which is different according to the wavelet-based function. Support-length is one of the important criteria for selecting the wavelet function. It is defined as the length that the wavelet-based function converges from a finite value to zero when the time or frequency tends to infinity [33]. The longer the support length is, the longer the computation time is. In order to simplify the calculation and fast convergence, a wavelet-based function with tight support and bi-orthogonality is usually selected. The following wavelets are commonly used:

(1) Haar wavelet. It is the earliest wavelet function with tightly supporting orthogonality used in wavelet analysis. It is defined as:

$$
\psi(t)=\left\{\begin{array}{lc}
1 & 0 \leq t \leq 0.5 \\
-1 & 0.5 \leq \mathrm{t} \leq 1 \\
0 & \text { other }
\end{array}\right.
$$

(2) Daubechies $(\mathrm{dbN})$ wavelet. The wavelet base in the Daubechies is denoted by the $\mathrm{dbN}$ function, where $\mathrm{N}$ is the order of the wavelet, which is a tightly supported standard orthogonal wavelet, with no explicit expression.

(3) Biorthogonal (biorNr.Nd) wavelet. The bi-orthogonal wavelet solves the contradiction between linear phase and orthogonality requirements. It uses one function to decompose and the other function to reconstruct, effectively taking into account the symmetry and accuracy of the signal.

(4) Symlets (symN) wavelet. The wavelet function is an improvement of the dbN function. Compared with the $\mathrm{dbN}$ wavelet, the symN wavelet has better symmetry, which can reduce the phase distortion during signal decomposition and reconstruction to a certain extent. 


\subsection{Normalization}

Normalization is a dimensionless treatment that makes the absolute value of a physical system value a relative value relationship. For the data to be normalized $x$, the normalization formula is as shown in Equation (7):

$$
x^{\prime}=\frac{\left(x_{\max }^{\prime}-x_{\min }^{\prime}\right) \cdot\left(x-x_{\min }\right)}{x_{\max }-x_{\min }}+x_{\min }^{\prime}
$$

where $x^{\prime}$ is the resulting normalized data, $x_{\max }^{\prime}$ and $x_{\min }^{\prime}$ are the maximum and minimum values of the predicted normalized data, usually pre-set, and $x_{\max }$ and $x_{\min }$ are the maximum and minimum values of the original data. The wavelet packet coefficients obtained by decomposing the wavelet packet are firstly normalized, and then the peak-type Markov chain modeling is used to construct a new wavelet coefficient, which greatly reduces the information loss of the wavelet packet coefficients in the modeling process. Therefore, the probability transfer matrix more accurately reflects the statistical characteristics of the wavelet packet coefficients, and finally improves the prediction accuracy of the noise.

\subsection{The Proposed Noise Model}

In this section, the wavelet peak-type Markov chain model is developed based on the methodology mentioned above to forecast the photovoltaic array channel noise which will be which will be described concretely in the next section; the basic structure of the model is given in Figure 7.

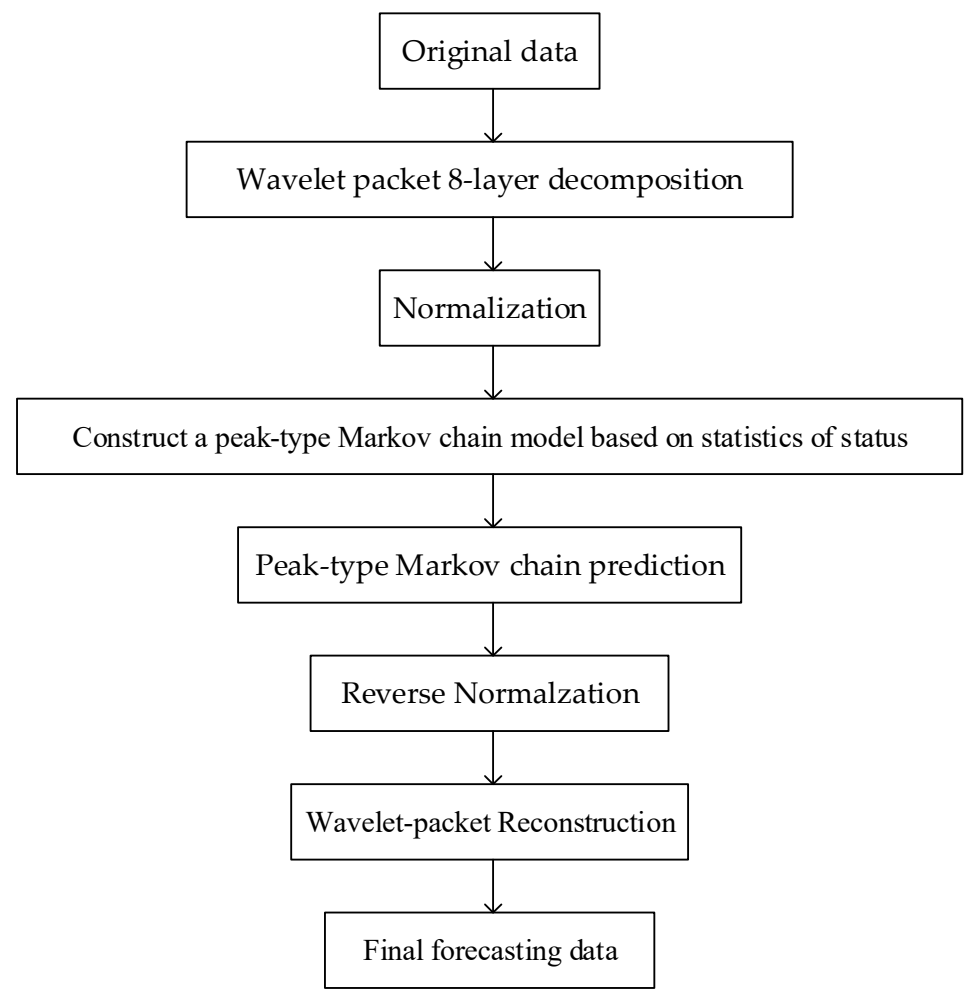

Figure 7. Basic structure of the proposed Wavelet peak-type Markov chain model.

Peak-type Markov chains can effectively model non-periodic pulse-type noise. The normalization algorithm can effectively prevent the loss of statistical information, which is essential for improving the accuracy of model prediction. Furthermore, the photovoltaic array channel noise is nonlinear and nonstationary time series data with relatively obvious volatility and periodicity, which can be handled by wavelet packet transform to some extent. Thus, the wavelet peak-type Markov chain model is 
established. It is necessary to note that, all calculating and modelling processes involved in this paper are realized in MATLAB 2014b in our study.

\section{Simulation and Results}

In this section, based on the measured photovoltaic array channel noise, not only the wavelet peak-type Markov chain model is established to study the applicability of the wavelet peak-type Markov chain model to the photovoltaic array channel noise, but also the influence of the selection of different wavelet-based functions on the prediction accuracy of the noise model. The modeling algorithm, as well as the simulation results, are presented.

\subsection{Modeling of Photovoltaic Array Channel Noise}

The collected noise signal is a time series containing 2500 sets of (time-noise amplitude) data. The specific modeling steps are as follows:

(1) Wavelet packet decomposition. The original noise sequence is decomposed into wavelet packet coefficients. Haar wavelet, Daubechies wavelet and Biorthogonal wavelet were respectively adopted to determine the best wavelet-based function when the photovoltaic array channel noise is modeled by wavelet peak-type Markov chain. It is necessary to note that the number of decomposition layer is 8 .

(2) Normalization. Conduct normalized preprocess on each set of wavelet packet coefficients.

(3) Statistics of status. Conduct status statistics on the normalized dimensionless data, then obtain the status transition matrices P1 and P2 of the rising and falling statuses respectively.

(4) Peak-type Markov modeling prediction. Based on the ascending status and the descending status transfer matrices, the peak-type Markov prediction is performed to obtain the new dimensionless data, which includes the information of the predicted wavelet packet coefficients.

(5) Reverse normalization. Denormalizing the dimensionless data to obtain a new wavelet packet coefficient.

(6) Wavelet packet reconstruction. The new wavelet coefficients are reconstructed to obtain a new noise sequence, layer by layer.

In order to verify the applicability of the wavelet peak-type Markov chain model to the noise of photovoltaic array channel, the modeling process of the noise which measured on experimental platform 1 and experimental platform 2 above are simulated respectively. To make the model have a relatively higher prediction accuracy, we study the influence of different wavelet-based functions on modeling accuracy first. Simulation results are presented in the following.

\subsection{Influence of Different Wavelet-Based Functions on Modeling Accuracy}

It is found that the different choice of wavelet-based function will have influence on the prediction accuracy of the wavelet peak-type Markov chain model.

Root-mean-square error (RMSE) is very sensitive to the extraordinarily large or small error in a set of measurements. It is widely used in engineering measurement, which is commonly used to describe the deviation between the forecast value and the true value. The expression is shown in Equation (8):

$$
R M S E=\sqrt{\frac{\sum_{i=1}^{n}\left(x_{i}-x_{i}^{\prime}\right)^{2}}{n}}
$$

Of which, $x_{i}$ is the $i$-th noise observation value, $x_{i}^{\prime}$ is the $i$ th noise model prediction value, and $n$ is the length of noise sequence. We use RMSE to measure the modeling effects of different wavelet-based functions. 
In order to determine the wavelet-based function with the best modeling consequence, this section takes the channel noise of the PV array measured in experimental platform 1 as the model object, and compares the accuracy of the prediction of the original noise when the Haar wavelet, Biorthogonal wavelet and Daubechies wavelet are respectively applied to the wavelet-based function for noise modeling. The Symlets wavelet is an improvement based on the symmetry of Daubechies wavelet. It has been experimentally found that the predicted waveforms under these two kinds of wavelets are almost the same, so this section does not take the Symlets wavelet into comparasion. In each case that wavelets above are respectively applied to modeling, the mean absolute error and root mean square error of time domain waveform points before and after modeling are calculated and compared.

Under the Windows 10 operating system working environment and MATLAB 2014b, the comparison of time-domain waveform points before and after modeling of each wavelet-based function are shown respectively in Figures 8-10, where it can be seen that the predicted noise by modeling with the three different wavelets all have good fitting with the original noise in the time-domain waveform, which proves the reliability of the modeling method above in some degree. Furthermore, the mean absolute error and root mean square error of the predicted output for each wavelet-based function modeling case are calculated, respectively.

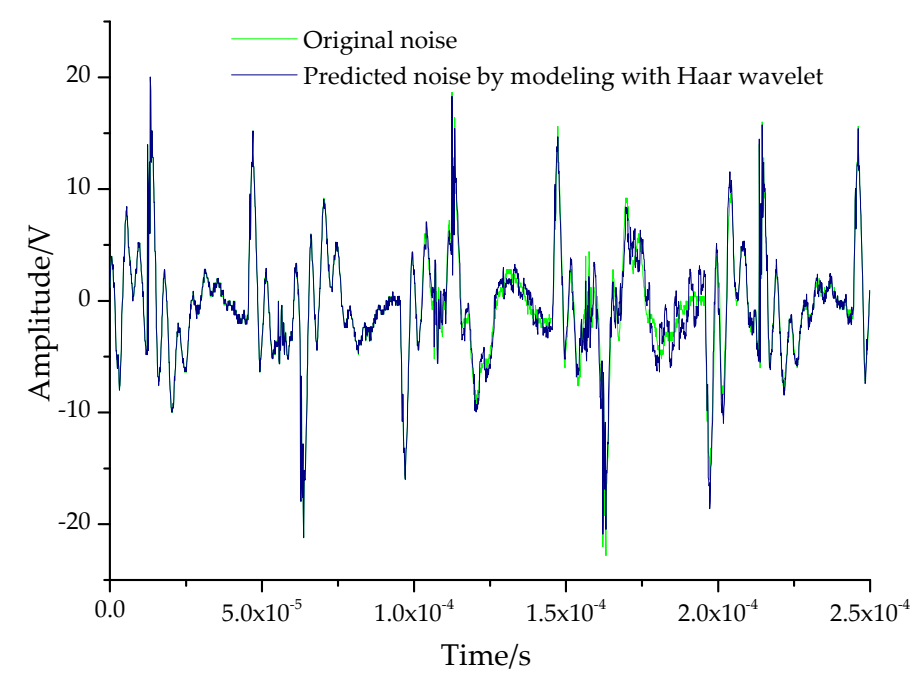

Figure 8. The comparison of time-domain waveforms before and after modeling with the Haar wavelet.

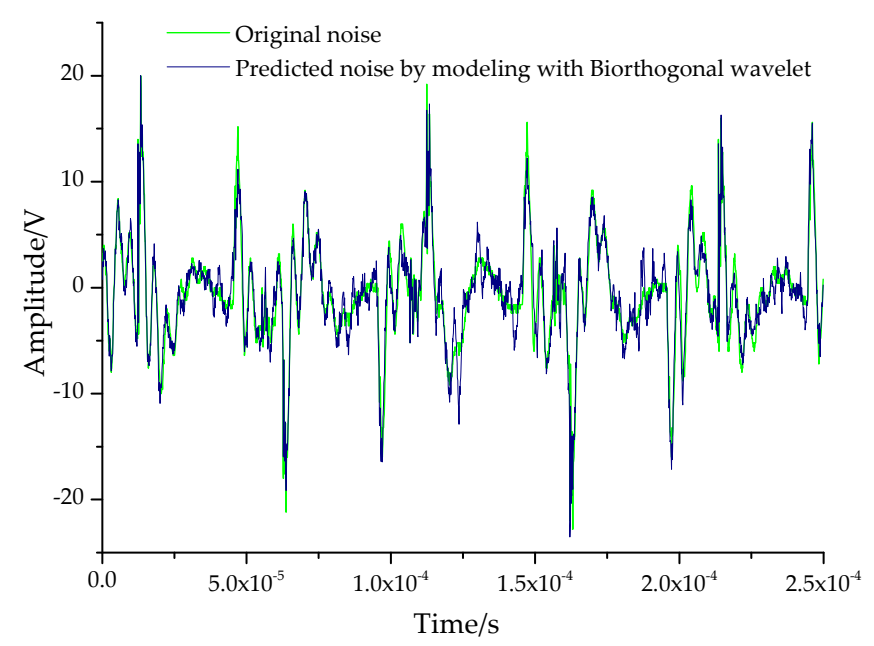

Figure 9. The comparison of time-domain waveforms before and after modeling with biorthogonal wavelet. 


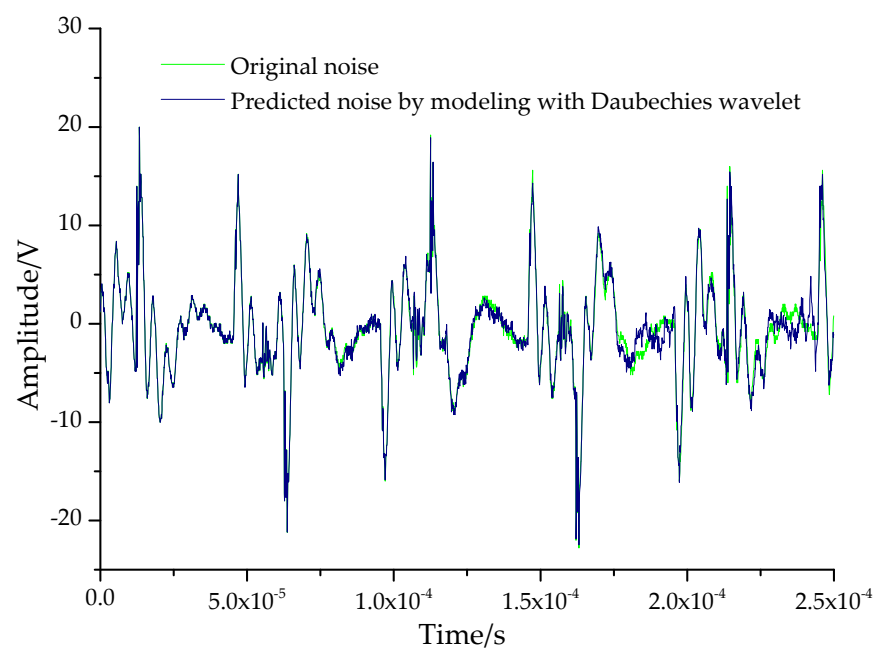

Figure 10. Comparison of time-domain waveforms before and after modeling with the Daubechies wavelet.

The results are shown in Table 1.

Table 1. The comparison of the predicted error for each wavelet-based function modeling case.

\begin{tabular}{ccc}
\hline Wavelet-Based Function & Mean Absolute Error/V & $\begin{array}{c}\text { Root Mean Square Error } \\
\text { (RMSE)/V }\end{array}$ \\
\hline Haar wavelet & 0.6097 & 0.9614 \\
Biorthogonal wavelet & 1.1381 & 1.4915 \\
Daubechies wavelet & 0.4926 & 0.7928 \\
\hline
\end{tabular}

The smaller the mean absolute error and the root mean square error, the higher the modeling accuracy. It can be seen from Table 1 that the Daubechies wavelet has the highest modeling accuracy, the Haar wavelet is the second, and the biorthogonal wavelet has a poorer modeling accuracy. From the comparison of the time-domain waveforms, it is seen that the predicted noise by modeling with Biorthogonal wavelet increases many high-frequency, small-amplitude background noise compared with the original noise, so the modeling with Biorthogonal wavelet has a poor modeling accuracy. The Haar wavelet modeling error is not much different from the Daubechies wavelet, but Haar is not continuously differentiable, the performance is not very good, and the application is limited. Inconclusion, the Daubechies wavelet is the best wavelet-based function when the photovoltaic array channel noise is modeled by wavelet peak-type Markov chain.

\subsection{The Simulation Results of the Proposed Noise Model}

This section presents the result of simulation, validating the applicability of wavelet peak-type markov chain model to photovoltaic array channel noise from the time domain characteristics and frequency domain characteristics. According to the study above, the Daubechie2 ( $\mathrm{db} 2)$ wavelet is adopted for the process of decomposition. The specific results of simulation are as follows:

\subsubsection{Simulation Results of Modeling the Noise Measured on the Experimental Platform 1}

Under the Windows 10 operating system working environment and MATLAB 2014b, the channel noise of the photovoltaic array measured on the experimental platform 1 is modeled. The comparison of part wavelet packet coefficient group obtained by peak-type Markov chain prediction and the original ones is shown in Figure 11. 

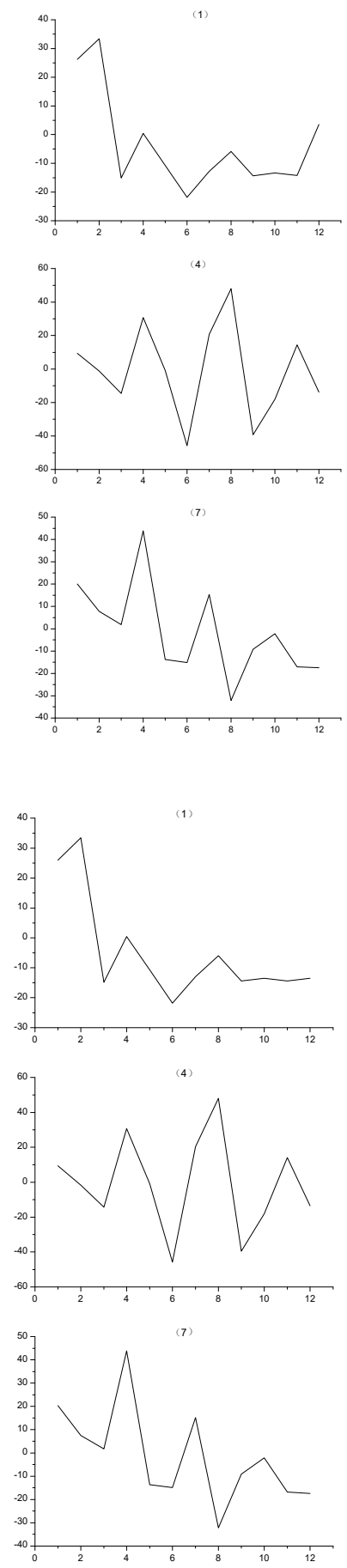
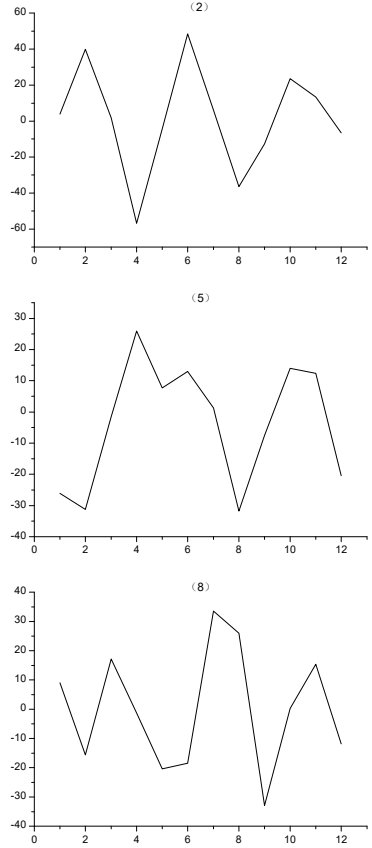

(a)
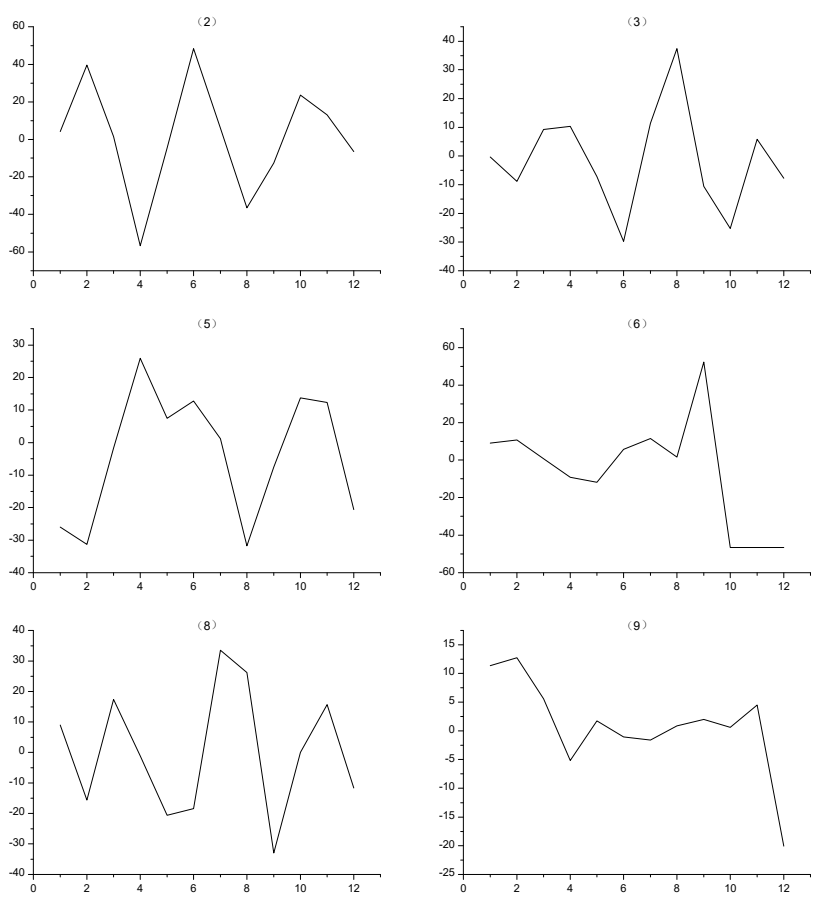

(b)

Figure 11. The part wavelet packet coefficient group before and after predicted. (a) Original part wavelet packet coefficient group; (b) Part wavelet packet coefficient group of peak-type Markov chain prediction.

The predicted new wavelet packet coefficient group and the original wavelet packet coefficient group have high fitting degree. The peak-type Markov chain can effectively predict the wavelet packet coefficient group whose amplitude exhibits non-periodic and pulse-type characteristic. The obtained new wavelet coefficient group is reconstructed to obtain a new noise sequence. The time-domain waveform and power spectral density (PSD) of the wavelet peak-type Markov chain output are shown in Figures 12 and 13. It can be seen from the figure that the time-domain waveform and the power 
spectral density trend of the forecast output are basically consistent with the original measured noise. The wavelet peak-type Markov chain model can effectively model the photovoltaic array channel noise measured in experimental platform 1 . The absolute error of the time-domain amplitude of the model output is obtained, and then the mean absolute error of the predicted output is $0.4926 \mathrm{~V}$.

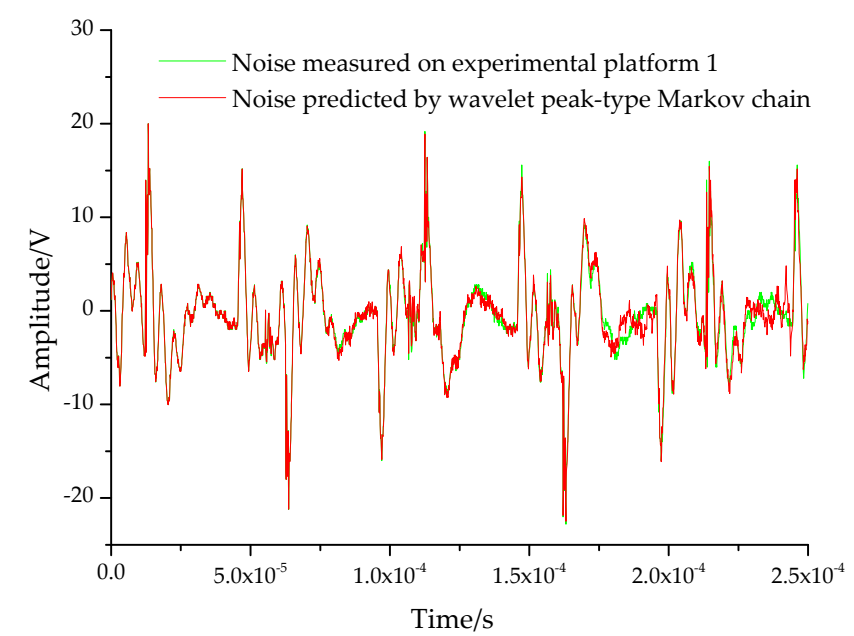

Figure 12. The time-domain waveforms of measured noise before and after modeling.

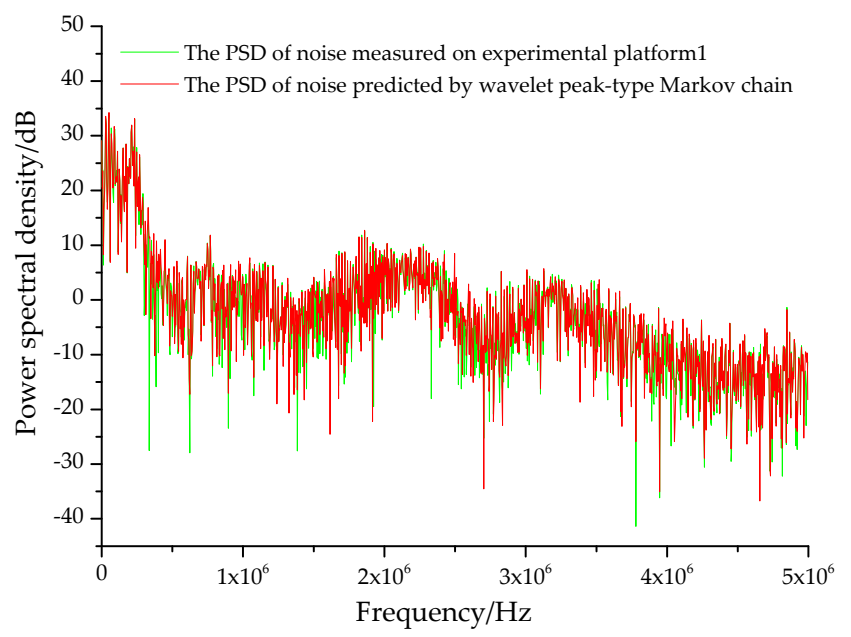

Figure 13. The power spectral density (PSD) of measured noise before and after modeling.

\subsubsection{Simulation Results of Modeling the Noise Measured on the Experimental Platform 2}

The same as the process of modeling PV array channel noise measured on experimental platform 1 , under the same environment, the channel noise of photovoltaic array measured on the experimental platform 2 is modeled. The comparison of part wavelet packet coefficient group obtained by peak-type Markov chain prediction and the original ones is shown in Figure 14. The predicted new wavelet packet coefficient group and the original wavelet packet coefficient group have high fitting degree, too. 

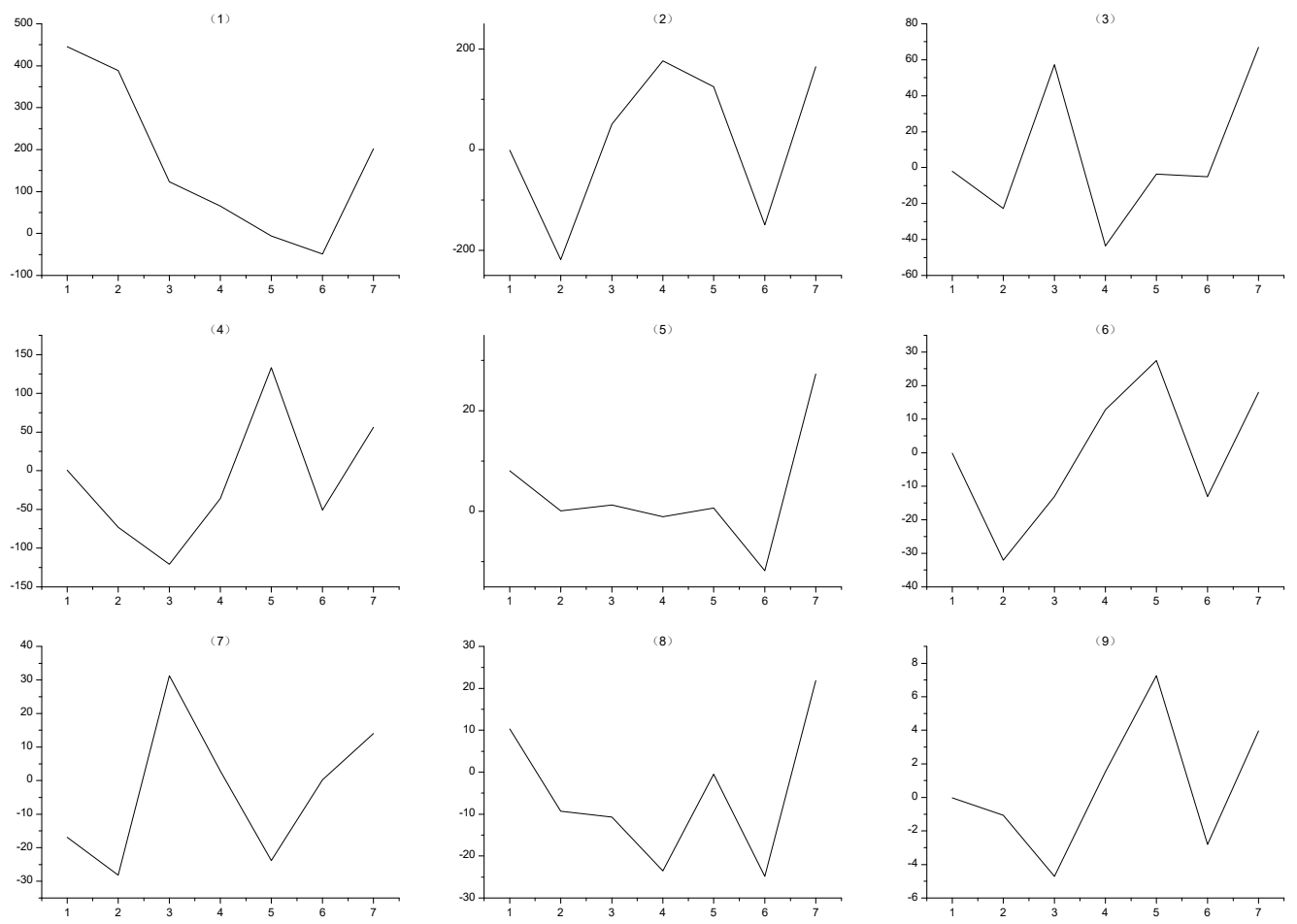

(a)
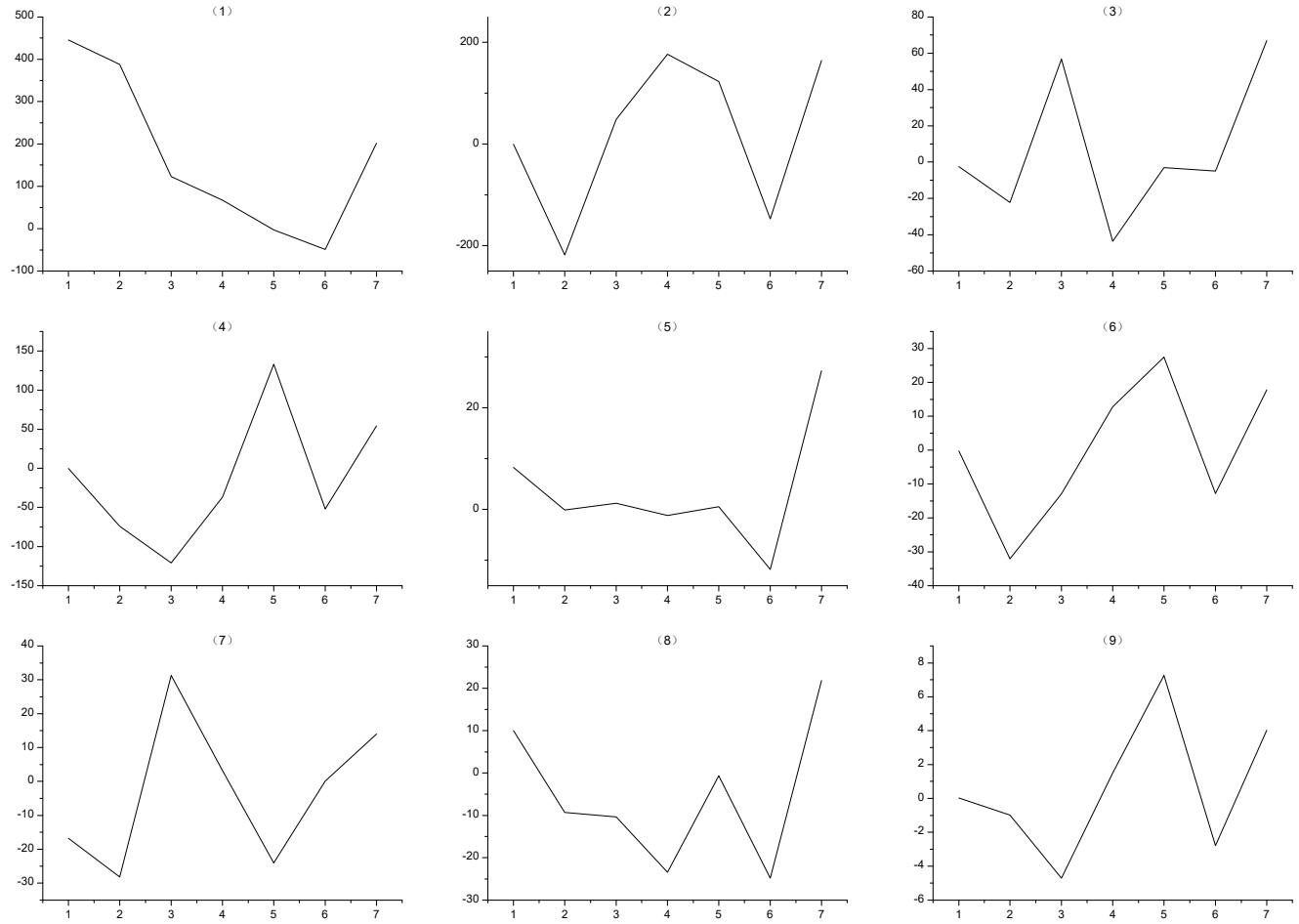

(b)

Figure 14. The part wavelet packet coefficient group before and after predicted. (a) Original part wavelet packet coefficient group; (b) Part wavelet packet coefficient group of peak-type Markov chain prediction.

The obtained new wavelet coefficient group is reconstructed to obtain a new noise sequence. The time-domain waveform and power spectral density (PSD) of the wavelet peak-type Markov chain 
output noise are shown in Figures 15 and 16. It can be seen from the figure that the time-domain waveform and the power spectral density (PSD) trend of the output are basically consistent with the original measured noise on the experimental platform 2. The mean absolute error of the wavelet peak-type Markov chain in predicting the amplitude of the noise measured on the experimental platform 2 is $0.6063 \mathrm{mV}$.

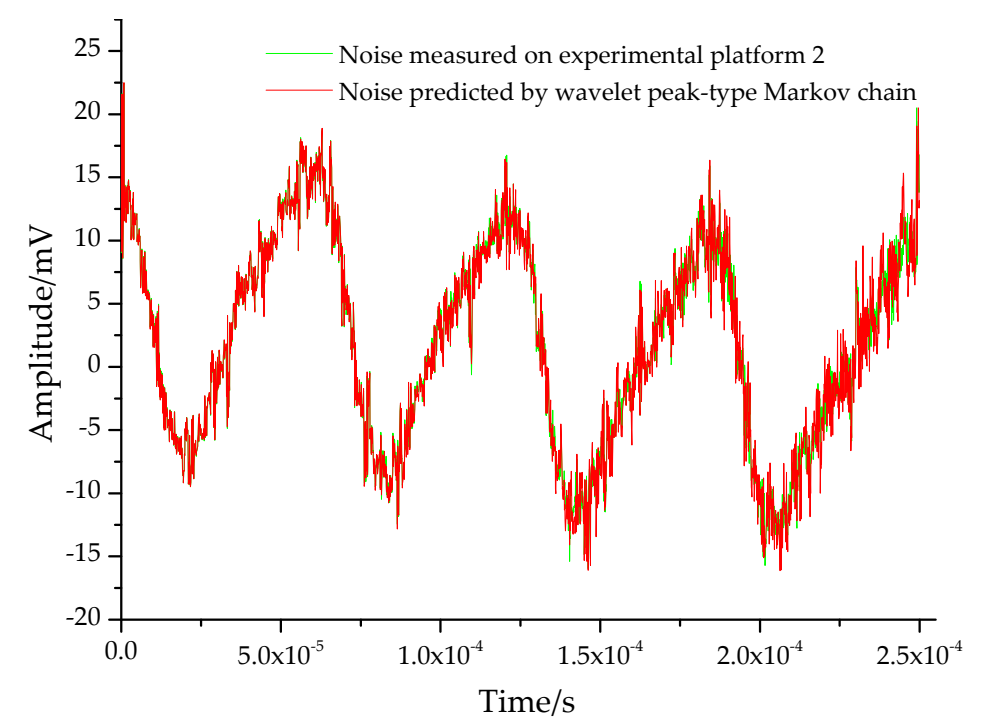

Figure 15. The time-domain waveforms of measured noise before and after modeling.

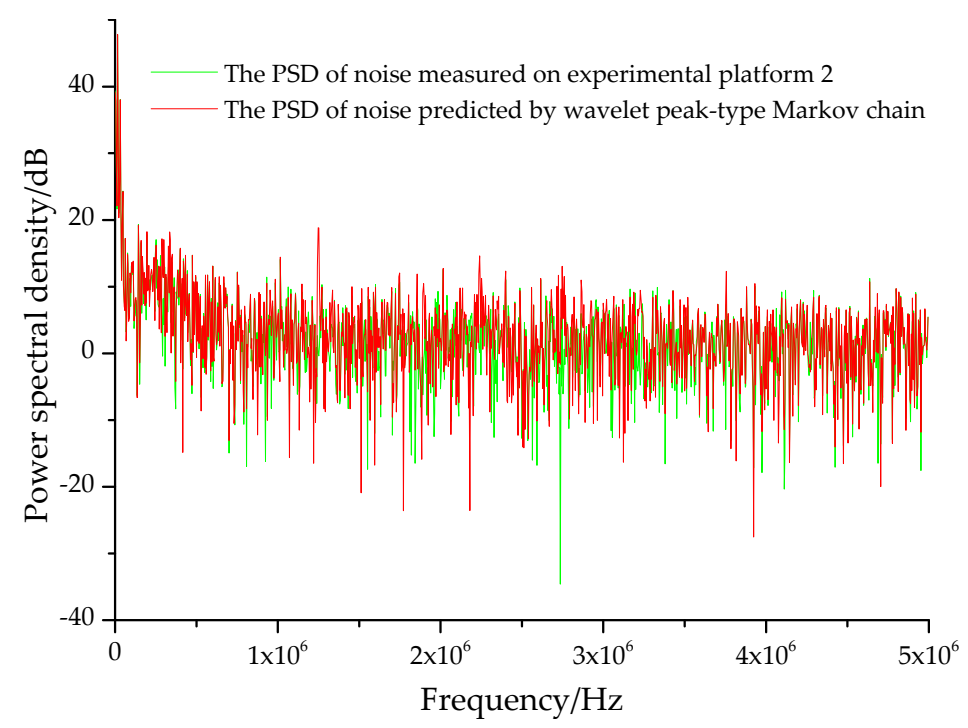

Figure 16. The power spectral density (PSD) of measured noise before and after modeling.

\subsubsection{Analysis of Prediction Error}

From the figures above, it is seen that the wavelet peak-type Markov chain can accurately model the photovoltaic array channel noise. The time domain characteristics and frequency domain characteristics of the output noise of the model are consistent with the original measured noise. The mean absolute error and root mean square error of the predicted time domain waveform points are both shown in Table 2. 
Table 2. The predicted error for modeling the noise measured in experimental platform 1 and 2.

\begin{tabular}{ccc}
\hline Experimental Platform & Mean Absolute Error (MAE) & Root Mean Square Error (RMSE) \\
\hline 1 & $0.4926 \mathrm{~V}$ & $0.7928 \mathrm{~V}$ \\
2 & $0.6063 \mathrm{mV}$ & $0.9693 \mathrm{mV}$ \\
\hline
\end{tabular}

In summary, the applicability of wavelet peak-type Markov chain model to photovoltaic array channel noise has been validated from the time-domain characteristics and frequency domain characteristics. The wavelet peak-type Markov chain noise model can accurately predict photovoltaic array channel noise.

\section{Conclusions}

Studying the channel noise characteristics of photovoltaic array is one of the prerequisites for using the PV array to realize carrier communication and providing the dynamic data of PV module operation to the power system big data center. In this paper, the causes of electromagnetic interference in photovoltaic series channel are analyzed. A photovoltaic array noise modeling method of wavelet peak-type Markov chain is proposed. Taking the measured noise in the experimental platforms of photovoltaic power stations as examples, the applicability of the modeling method to the photovoltaic array channel noise is studied by simulation. The effects of different wavelets on modeling accuracy are studied, too. The main novel conclusions are as follows:

(1) After measurement, there are a large number of periodic spike noises with high amplitude in the photovoltaic array channel. These noise interference signals are mainly generated by photovoltaic modules and inverters, and after analysis, voltage and current type interference signals coexist, and differential-mode interference and common-mode interference coexist. In order to better explain the existence of electromagnetic interference (EMI), a simplified EMI equivalent circuit model of the photovoltaic power generation system has been constructed.

(2) The simulation results show that selecting the criteria of wavelet support function with tight support and bi-orthogonality, among the Haar wavelet, biorthogonal wavelet and Daubechies wavelet, the Daubechies wavelet as the wavelet-based function for modeling that has the highest fitting precision, which makes it the best wavelet function for modeling photovoltaic array channel noise.

(3) The simulation results also show that the wavelet peak-type Markov chain can accurately model the photovoltaic array channel noise. Both time-domain characteristics and frequency-domain characteristics of the output noise forecast by the model are consistent with the original measured noise. In the case that the peak of original noise reaches $20 \mathrm{~V}$, the predicted mean absolute error of this model is only $0.4926 \mathrm{~V}$, which not only verifies the applicability of the Wavelet Peak-Type Markov chain model to the photovoltaic array channel noise, but also verifies the applicability to the pulse-type noise.

Author Contributions: For all authors contributed equally to this work. F.S. proposed the original idea. C.Z. designed the overall framework of this paper, conducted data analysis, built the simulation model, completed the manuscript, modified and refined the manuscript. All authors read and approved the final manuscript.

Funding: This research is supported by the Fundamental Research Funds for the Central Universities (2016MS06).

Acknowledgments: This study was conducted as part of the project cooperated with the State Grid Corporation of Qinghai, China.

Conflicts of Interest: The authors declare no conflict of interest.

\section{References}

1. Sun, J.M.; Sun, F.J.; Fan, J.Q.; Liang, Y.T. Fault Diagnosis Model of Photovoltaic Array Based on Least Squares Support Vector Machine in Bayesian Framework. Appl. Sci. 2017, 7, 1199. [CrossRef]

2. Qiao, L.; Liu, Y.; Liu, W. Probe into the Application of Power Big Data in Smart Grid. Electron. World 2019, 0, 36-37. [CrossRef]

3. Zhang, D.X.; Miao, X.; Liu, L.P.; Zhang, Y.; Liu, K.Y. Research on Development Strategy for Smart Grid Big Data. Proc. CSEE 2015, 35, 2-12. [CrossRef] 
4. Zhou, Y.L.; Liu, X. Discussion on Big Data Processing Technology of Smart Grid. Electron. World 2019, 0, 180-181. [CrossRef]

5. Xie, Z.Y.; Chen, N.; Zhang, D.Q. Design of leakage monitoring system for photovoltaic power station based on power line communication. Appl. Electron. Tech. 2018, 44, 40-42, 46. [CrossRef]

6. Meng, L.B. Research and Application of Carrier Communication Technology in Monitoring System of Photovoltaic Power Station. Master's Thesis, Fuzhou University, Fujian, China, June 2013.

7. Yang, F. Photovoltaic Module Monitoring System Based on DC Power Line Carrier Communication. Master's Thesis, Nanjing University of Posts and Telecommunications, Nanjing, China, March 2017.

8. Zhang, J.H. The Design of Wireless Monitoring System for Photovoltaic Power Station Based on CC2530. Master's Thesis, Xian University of Technology, Xian, China, June 2016.

9. Zhang, X.Y.; Xie, R.; Zeng, J. Photovoltaic power station wireless monitoring system based on component information measurement. Sol. Energy 2016, 0, 40-43, 47. [CrossRef]

10. Sun, F.J.; Bao, J.J.; Wang, D.F.; Yang, G. Research on DC Single-conductor Carrier Communication Technology between Photovoltaic Modules. In Proceedings of the 2017 Smart Grid Development Seminar, Beijing, China, 6-8 June 2017; p. 5.

11. Su, N.; $\mathrm{Xu}, \mathrm{D}$. Analysis and verification of ground current for grid connected transformerless photovoltaic inverter system. Autom. Electr. Power Syst. 2012, 36, 30-35.

12. Wu, M.; Song, Z.H.; Lv, Z.P.; Zhou, K.; Cui, Q. A Method for the Simultaneous Suppression of DC Capacitor Fluctuations and Common-Mode Voltage in a Five-Level NPC/H Bridge Inverter. Energies 2019, 12, 779. [CrossRef]

13. Guo, X.Q.; Wei, B.Z.; Yang, Q.X.; Zhao, Q.L.; Wang, B.C.; Sun, S.F. Leakage current suppression method with a novel six-switch photovoltaic grid-connected inverter. Autom. Electr. Power Syst. 2014, 38, 101-105. [CrossRef]

14. Zhang, J.; Chen, W.J.; Zhang, B.; Yang, X. DC side EMI filter analysis in transformer-less inverter. Trans. China Electrotechnical Soc. 2015, 30, 107-112. [CrossRef]

15. Duan, Z.L.; Fan, T.; Zhang, D.; Wen, X.H. Modeling and prediction of electromagnetic interference in whole SiC three phase inverters. Adv. Technol. Electr. Eng. Energy 2018, 37, 1-7. [CrossRef]

16. Shi, P.X. Modeling and Prediction of Conduction Electromagnetic Interference in PWM Inverter. Master's Thesis, Huazhong University of Science and Technology, Wuhan, China, May 2017.

17. Zhou, Z.J. Research on the Modeling and Reduction of Conducted Electromagnetic Interference in Inverter Power System. Master's Thesis, Huazhong University of Science and Technology, Wuhan, China, May 2017.

18. Ye, J.; Sun, H.L.; Wang, Y.; Wen, C.L. Modeling method of background noise in power line communication channel. J. Chongqing Univ. Posts and Telecommun. (Nat. Sci. Ed.) 2015, 27, 722-727.

19. Li, B.; Mauro, T.; Sami, B.; Marco, R.; Tao, Z. Impulsive Noise Characterization in Narrowband Power Line Communication. Energies 2018, 11, 863. [CrossRef]

20. Ying, Z.F.; Wu, J.J.; Guo, H.K.; Wan, M. Modeling of low-voltage power line noise containing periodic pulses. Electr. Power Autom. Equip. 2013, 33, 58-63. [CrossRef]

21. Li, C.L. Modeling Research of Noise in Power Line Based on Wavelet-Markov Chain. Master's Thesis, Nanjing University of Science and Technology, Nanjing, China, March 2014.

22. Xiao, J.W. Research on Conducting Electromagnetic Interference Technology of Photovoltaic Off-Grid Inverter System. Master's Thesis, Nanjing Normal University, Nanjing, China, March 2013.

23. Jing, L.; Yang, J.; Wu, X.Z.; Tong, Y.B. Analysis of common mode voltage between parallel photovoltaic inverters DC-link. Acta Energiae Solaris Sinica 2014, 35, 2106-2112. [CrossRef]

24. Fahrenbruch, A.L.; Bube, R.H. Fundamentals of Solar Cells; Academic Press: New York, NY, USA, 1983.

25. Pei, X.J. Research of Conducted Electromagnetic Interference in PWM Inverter. Ph.D. Thesis, Huazhong University of Science and Technology, Wuhan, China, September 2004.

26. Chen, Y.A.; Zhang, W.P. Expanded frequency measurement of frequency characteristic of Al electrolytic capacitor by means of Agilent4395A. China Meas. Technol. 2007, 33, 44-46. [CrossRef]

27. Guo, H.K. Modeling Research of Noise in Low-Voltage Power Line Communication Channel. Master's Thesis, Nanjing University of Science and Technology, Nanjing, China, March 2013.

28. Feng, K.; Ying, Z.F.; Wu, J.G.; Wan, M. Short-term wind speed forecast based on wavelet packet decomposition and peak-type Markov chain. J. Nanjing University Sci. Technol. 2014, 38, 639-643. [CrossRef] 
29. Wang, H. Study of Short-term Load Forecasting Based on Wavelet Packet Analysis. Master's Thesis, Nanjing University of Science and Technology, Nanjing, China, March 2013.

30. Chen, J.H.; Zou, S.L. An Intelligent Condition Monitoring Approach for Spent Nuclear Fuel Shearing Machines Based on Noise Signals. Appl. Sci. 2018, 8, 838. [CrossRef]

31. Wang, X.; Shi, T.L.; Liao, G.L.; Zhang, Y.C.; Hong, Y.; Chen, K.P. Using Wavelet Packet Transform for Surface Roughness Evaluation and Texture Extraction. Sensors 2017, 17, 933. [CrossRef] [PubMed]

32. Sahar, Z.; Samsul, B.M.N.; Mohammad, R.M.; Mohammad, H.M.; Norman, M. Broken Rotor Bar Fault Detection and Classification Using Wavelet Packet Signature Analysis Based on Fourier Transform and Multi-Layer Perceptron Neural Network. Appl. Sci. 2018, 8, 25. [CrossRef]

33. Suo, C.N.; Zhang, H.; Zhao, X.W. Research on the application of wavelet basis functions in modeling of colored background noise for low-voltage power line channels. Power Syst. Prot. Control 2017, 45, 121-125. [CrossRef]

(C) 2019 by the authors. Licensee MDPI, Basel, Switzerland. This article is an open access article distributed under the terms and conditions of the Creative Commons Attribution (CC BY) license (http://creativecommons.org/licenses/by/4.0/). 